\title{
Efectos de las estrategias empresariales de los AGENTES BANPRO en la SATISFACCION DE LOS CLIENTES en la ciudad de Estelí en el año 2014 ${ }^{1}$
}

\author{
José Miguel González Navarrete ${ }^{2}$ \\ Keylin Haritza Ruíz Reyes ${ }^{3}$ \\ Mileyda Lisseth Ruiz González ${ }^{4}$ \\ Beverly Castillo Herrera ${ }^{5}$
}

\begin{abstract}
RESUMEN
El presente trabajo determina los efectos de las estrategias empresariales de los agentes BANPRO para diseñar propuestas que mejore la satisfacción de los clientes y agentes en la ciudad de Estelí en el año 2014. El desarrollo de este estudio se hizo tomando en cuenta el enfoque cualitativo. El universo de estudio fue 23 agentes los cuales se encuentran ubicados en el municipio, con un tipo de muestreo no probabilístico porque los sujetos de consulta son elegidos en base a criterios selectivos como: Propietarios de establecimientos bancarios, clientes de BANPRO que usan este servicio, disponibilidad de las personas en brindar la información.
\end{abstract}

Los instrumentos utilizados en el estudio fue la entrevista, guía de observación y por carácter del segundo objetivo se aplico encuestas dirigidas a clientes. Una limitante para el crecimiento de estos agentes es la publicidad muchos clientes desconoce este servicio y es inevitable su desconfianza al utilizarlo por primera vez, otra es la ubicación del POS dentro del local porque no garantiza la seguridad del cliente, haciendo que los usuarios recurran a las sucursales bancarias para realizar sus pagos.

Es fundament al que BANPRO diseñe planes estratégicos para mejorar las limitantes que tienen como diseñar ventanillas para estos establecimientos y la importancia de invertir en publicidad para dar a conocer lo que se oferta.

Palabras claves: Banco, BANPRO, Estrategia Empresariales, Satisfacción de clientes, Estelí

1 Este artículo se basa en la investigación titulada Efectos de las estrategias empresariales de los AGENTES BANPRO en la SATISFACCION DE LOS CLIENTES en la ciudad de Estelí en el año 2014. Para obtener el título de Licenciada en Administración de Empresas de la UNAN-Managua, FAREM Estelí.

2 UNAN-Managua FAREM-Estelí, Correo electrónico: gonzalezn.josem@yahoo.com

3 UNAN-Managua FAREM-Estelí, Correo electrónico: reyes.keling10@yahoo.com

4 UNAN-Managua FAREM-Estelí, Correo electrónico: miley.ruiz@yahoo.com

5 Candidata a doctora en Ciencias Sociales por la Universidad del Zulia. Maestría en Ciencias Sociales por la Universidad de Guadalajara. Docente titular de la UNAN-Managua, FAREM-Estelí. Correo Electrónico: beverly.castillo@yahoo.com. 


\title{
Effects of business strategies of BANPRO AGENTS in CUSTOMER SATISFACTION in the city of Estelí in 2014
}

\author{
José Miguel González Navarrete \\ Keylin Haritza Ruíz Reyes \\ Mileyda Lisseth Ruiz González \\ Beverly Castillo Herrera
}

\begin{abstract}
The present study determines the effects of corporate strategies of BANPRO Agents (Bank of Production) to design proposals to improve customer satisfaction and agents' service in the city of Estelí in 2014. This study is qualitative. The study group was 23 agents located in the city of Estelí. The sampling was non-probability, considering the following criteria: owners of banking establishments, customers using this service, and availability of people to provide information. Interviews, surveys and observation guides were applied.

This study shows that the lack of publicity is one of the main constraints for the growth of BANPRO agents since many customers are unaware of this service and it is inevitable their distrust when using it for the first time. Another limitation is the location within the buildings because it does not ensure customer safety. It is recommended that the bank designs strategic plans to improve the constraints they have such as to design windows for these establishments, and the importance of investing in advertisement to make notorious what it is offered.
\end{abstract}

Keywords: Bank, BANPRO, Business Strategy, Customer Satisfaction, Estelí 


\section{INTRODUCCIÓN}

En los últimos años, multitud de empresas de diversos sectores económicos han adoptado tecnologías de la información y la comunicación como el teléfono, Internet o los teléfonos móviles, con ello, se han lanzado a la conquista de nuevos espacios, creando, sin saberlo, nuevos canales de comunicación con los clientes, logrando la plena "satisfacción del cliente" siendo requisito indispensable para ganarse un lugar en la "mente" de los clientes y por ende, en el mercado meta.

Con la visión hacia un futuro BANPRO como entidad bancaria se ha diferenciado de su competencia en brindar nuevos canales de interacción con los clientes sin que estos visiten la sucursal.

La presente investigación se enfoca en el servicio que ofrece el Banco de la Producción, que son los "Agentes BANPRO". Como un nuevo canal de atención que permite a los clientes realizar diversas transacciones bancarias, ubicadas en negocios comerciales en los diferentes barrios de la ciudad de Estelí y serán plenamente identificados con la marca institucional del Banco.

Los objetivos de los Agentes Bancarios se enfocan en: Bancarización a pequeños y medianos comercios, atender a los clientes del Banco de la producción de una forma conveniente, sin visitar una sucursal tradicional, así también ampliar la cobertura del servicio del Banco de la producción.

Las transacciones que se puede realizar en los Agentes BANPRO incluye: Consulta de saldo, retiros, depósitos, pago de tarjeta de crédito, venta de tiempo aire, pago de servicios básicos, remesas y pagos de financiamientos.

La transcendencia de la investigación beneficia al Banco para analizar el desarrollo de este nuevo servicio y las problemáticas que posiblemente han ocurrido, para darse cuenta si los objetivos propuestos se han logrado y plantear nuevas estrategias para su crecimiento y aceptación del servicio por los clientes.

Entre los principales limitantes que tiene este servicio son: la publicidad que se le da a los agentes BANPRO no es la adecuada para la compresión del usuario, para que este se sienta en confianza al usarlo, la seguridad a la hora de realizar la operación ya que no existe un espacio adecuado dentro del local para realizarla, los problemas con el sistema para realizar pagos de servicios básicos (agua, luz, teléfono), la necesidad de reimpresión del voucher por problemas que se presente, y la incorporación de otros pagos y servicios que se pueda realizar.

Esta investigación beneficia a los estudiantes para adquirir nuevos conocimientos sobre el manejo estratégico que el banco utiliza para satisfacer y atraer a clientes actuales y nuevos. A los agentes bancarios para implementar estrategias partiendo desde los problemas que se han observado detalladamente, y a la sociedad para brindarle conocimientos necesarios para que utilice el servicio y sea de confiabilidad y de facilidad para el usuario.

Es de gran importancia el estudio de los Agentes BANPRO, ya que se va conociendo la realidad del campo profesional y los problemas que estos enfrentan al lanzar al mercado un nuevo producto o servicio.

Entre los ejes teoricos de la investigación son:

Bancos son las instituciones financieras autorizadas como tales, dedicadas habitualmente a realizar operaciones de intermediación con recursos obtenidos del público en forma de depósitos a cualquier otro título, y a prestar otros servicios financieros. (Blandón García, Sergio (2008). Pág. 3). 
La estrategia empresarial es la determinación de la posición futura de la empresa, en especial frente a sus productos y mercados, su rentabilidad, su tamaño, su grado de innovación y sus relaciones con sus ejecutivos, sus empleados y ciertas instituciones externas. Esta posición estratégica debe equilibrarse mediante la planeación estratégica de la empresa partir de tres actividades básicas (Chiavenato. I. 2002. pág. 116).

Los consumidores intercambian recursos, normalmente en forma de dinero, por los productos y servicios de la organización. Un cliente puede ser una institución, pro ejemplo una escuela, hospital u oficina de gobierno; puede ser otra empresa, por ejemplo un contratita, distribuidor o fabricantes; o una persona física. Por regla general, un gerente de mercadotecnia analiza a los consumidores potenciales, así como las condiciones de mercado, y dirige una campaña de mercadotecnia con base en dicho análisis. (Stoner. J. Freeman. E. Gilbert. D. 1996. Pág. 296).

Satisfacción de los clientes es la percepción que tiene el individuo sobre el desempeño del producto o servicio en relación con sus exceptivas. Los consumidores tendrán expectativas bastante diferentes cuando visitan un restaurante francés caro y cuando visitan McDonald's aunque ambos formen parte de la industria restaurantera. El concepto de satisfacción de consumidor es una función de las expectativas del cliente. Un consumidor cuya experiencia este por debajo de sus expectativas (como recibir frías las papas fritas en un McDonald's o cuando los platos usados no se retiran con la prontitud suficiente en un restaurante caro) quedaran insatisfecho. (Schiffman. L. Kanuk. L. 2005. Pág. 14).

\section{MATERIALES Y MÉTODOS}

Para llevar a cabo el estudio se utilizaron fuentes primarias de carácter cualitativo cuyo instrumento fueron principalmente la entrevista y guía de observación porque se profundiza sobre el estudio de caso. Se aplicó tres entrevistas semi-estructuradas dirigidas una a los diferentes establecimientos Agentes BANPRO, el Banco y los clientes, así como las encuestas a los usuarios del nuevo servicio. Se realizó durante todo el mes de Junio- 2014. Para el éxito de esto se hizo un proceso de inducción a cada uno de los participantes de entrevistas y encuestas explicándoles cual era el objeto de la investigación y solicitándoles su colaboración, las entrevistas duraron un período de 15 a 25 minutos.

Se aplicaron 18 entrevistas en los diferentes establecimientos BANPRO, 25 entrevista clientes y una entrevista a Alcides Ramó Ruíz Técnico de los agentes BANPRO. Se aplicaron 360 encuestas en las áreas geográficas localizadas cerca de los agentes BANPRO ubicados en la ciudad de Estelí. De igual manera se aplicó una guía para conocer el entorno a este nuevo servicio de agentes BANPRO.

También se hizo una revisión bibliográfica de diferentes libros y trabajos de curso para enriquecer y dar veracidad a la información obtenida durante esta investigación.

\section{RESULTADOS Y DISCUSION}

\section{Antecedente de BANPRO}

El Banco de la Producción, S.A. (Banpro) es una sociedad privada fundada el 11 de noviembre de 1991, con el propósito de intermediar depósitos del público para invertirlos en una amplia variedad de negocios de los sectores productivos y de servicios, para servir como agente de desarrollo al país.

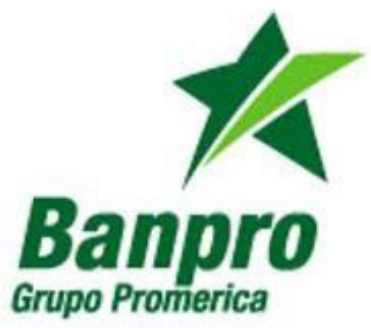


De los bancos que hoy en día forman parte del Sistema Financiero de Nacional, Banpro fue el primer banco en abrir sus puertas en Nicaragua cuando en el país se reiniciaron las condiciones para el desarrollo de la inversión privada. Fue inaugurado con el aporte de 133 socios de las más diversas actividades productivas del país.

En el transcurso de todos estos años desde su fundación, BANPRO Grupo Promerica ha experimentado un crecimiento sostenido de sus activos, de los depósitos que se captan del público y de su patrimonio, hasta convertirse en el banco más grande de la nación, y en relación a los bancos de América Central y su tamaño se ubica dentro de los 20 más grandes en cuanto a activos se refiere. Para lograr esta posición privilegiada Banpro ha desarrollado una estrategia de crecimiento basada en servicio con valor agregado a los clientes.

Actualmente el Banco de la Producción S.A cuenta con más de 70 sucursales alrededor de Nicaragua para garantizar mejores condiciones para los clientes.

Sus principales productos son: las tarjetas de créditos, cuentas, préstamos. Entre los servicios: asesoria, caja de seguridad, etc. BANPRO es una gran empresa, cuenta con un numero de (250-1000 trabajadores).

\section{Agente BANPRO}

Constituye un nuevo canal de atención de BANPRO, que permite a los clientes realizar diversas transacciones bancarias, a través de negocios comerciales ubicados a nivel nacional y identificado con la marca institucional del banco.

\section{Los objetivos de los Agentes BANPRO}

La bancarización a pequeños y medianos comercios, atender a los clientes de una forma conveniente, sin visitar una Sucursal tradicional, y ampliar la cobertura de Servicio de Banco de la Producción.

\section{Las transacciones Soportada de los Agentes son:}

Pago de financiamiento, consulta de saldo, retiros, depósitos, pago de tarjeta de crédito, venta de tiempo aire y remesas.

\section{Ventaja de los Agentes BANPRO para los clientes}

- Conveniencia: Más puntos de atención, reduciendo tiempo y costo de traslado.

- Economía: Transacciones libres de comisiones.

- Accesibilidad: Mayor horario de atención ajustándose al del establecimiento.

- Atención “cara a cara": Más amigable y cercana que otros medios electrónicos

- Elimina la necesidad de trasladarse a localidades donde existen instituciones financieras

\section{Ventaja de los Agentes BANPRO para los comercio}

- Aumenta la oferta de servicios para sus clientes.

- Tráfico: Incrementa número de personas que ingresan al comercio; posibilitando la venta cruzada y mayores ingresos.

- Diferenciación: Mejora imagen y prestigio al ser aliada de una acreditada institución financiera del país.

- Compensación económica: Recibe una comisión según el número de transacciones que atienda por día.

\section{Desventaja de los Agentes BANPRO para los comercio}

- Competencia del Banco y la Ventanilla.

- Competencia de los demás agentes BANPRO.

- No se puede realizar todas operaciones que se hacen en la entidad financiera.

- Problema en cargar algunos servicios (Luz, Agua),

- Reimpresión del voucher.

- Publicidad no concreta sobre este nuevo servicio 


\section{Operaciones del Agente BANPRO}

El horario de Atención la establece el negocio, el personal que utiliza este sistema es capacitacitado en el manejo del mismo, BANPRO provee del material publicitario para la identificación del comercio de parte del Banco, todas las transacciones de agente (efectivo y tarjeta) se computarán a través de los pos y al momento del cierre se imprimirá el detalle de las operaciones por lote del POS.

Limites en efectivo no mayores a $C \$ 4,000.00$ por transacción Por el valor máximo para realizar la transacción dicen: El monto no ha sido inconveniente, ya que se ha presentado el caso en que personas exceden el monto de 4000 y simplemente se divide uno, dos o tres transacciones y genera más ganancias al agente, pero es algo que no se da mucho para no atrasar al cliente. (Ramiro Figueroa, Dueño de distribuidora Figueroa, Entrevista 16 de Junio del 2014).

Por lo que el monto máximo de transacción no ha sido inconveniente en mi negocio, por tanto los clientes siempre se van satisfechos y a gusto con el servicio. (Dueña de la pulpería JEAR, entrevista 15 de Junio 2014).

Existe un monto que establece el Banco a inicio que es de 4000 pero no tengo un monto establecido de transacción ya que va en dependencia de la disponibilidad de dinero que tenga y en mi negocio se hacen transacciones mayores al monto de 4000. (Encargado en la Ferretería Palace, entrevista 16 de Junio 2014)

\section{Valoración de los cliente que usan los Agentes BANPRO}

Se aplicó una encuesta donde obtuvo que el 59.44\% de los clientes que utilizan el nuevo servicio agentes BANPRO son del sexo femenino y el $40.56 \%$ de los clientes son del sexo masculino lo que quiere decir que estos negocios son comúnmente más visitados por mujeres.

Los principales clientes que utilizan el nuevo servicio agentes BANPRO se encuentra entre un rango de edad de 20 a 30 años, con un porcentaje de $46.39 \%$ representado la mitad de las personas que visitan estos lugares. Un propietario de estos establecimientos comento "En mi establecimiento los jóvenes frecuenta más ya que la mayoría bien a pagar servicio básico y a compra de tiempo aire siendo esto el motivo de sus visitas". (Don Julio Gutiérrez - Seudónimo, Entrevista 15 de Julio del 2014).

En segundo lugar ocupa un 27.22\% las personas en un rango de edad de 31 a 40 años. Siguiendo con un $21.67 \%$ las personas entre 41 a 50 años (Doña Flor Zeledón-Seudónimo, Entrevista 15 de Julio del 2014) dueña de uno de lo establecimiento Comento: "las señoras cuando viene hacer sus compras para cocinar aprovecha a pagar los recibos".

\section{Estrategia empresariales de los Agentes BANPRO}

De los 360 asociados en la encuesta 159 de ellos representado el $35.57 \%$ nos comentaron que la publicidad por la cual ellos se dieron cuentan de la existencia de los agentes BANPRO, es la que ha hecho los propios propietarios de los negocios ya que el banco no ha enfatizado en este punto. (Ramón Canales, Propietario de la Pulpería Olga- Entrevista 16 Junio del 2014). En la visita que se nos comentó que elaboraron sus propios volantes publicitarios reflejando cuales son las transacciones que se pueden hacer en su establecimiento. Así como la información genera de su establecimiento.

Una cliente comento "si no es por ellos que nos dicen sobre ese servicio no nos diéramos cuenta de su existencia" (Doña Lourdes López- Seudónimo, Entrevista 15 de Junio del 2014). El 33.56\% que equivale a 150 personas la publicidad por el cual 
se dieron cuenta es por el comercial en televisión de los agentes BANPRO, un señor comento "vi el comercial pero no es muy claro sobre este servicio ya que sale un señor con otro hablando sobre esto, pero no refleja nada”. El 11.41\% que son 51 personas respondieron la radio que ellos escucharon que uno de los establecimientos se anunciaba en este medio. El $50 \%$ se dieron cuenta de los Agentes BANPRO por los volantes un joven nos comentó "la pulpería Olga elaboro sus propios volantes lo cual es innovador ya que utiliza publicidad para que su establecimiento sea frecuentado". (Julio Martínez- Seudónimo, Entrevista 17 de Junio del 2014).

La estrategia que estos propietarios emplearon comentaron: Como agente no utilizo ninguna estrategia para atraer a los clientes ya que el porcentaje que me genera el banco es tan bajo que no me es de mucho interés debido a que la ferretería me genera mucho más dinero y la única estrategia para atraer a los clientes es la genera el banco que son afiches, banners y volantes de publicidad. (Dueño de Ferretería Estelí, entrevista 17 de Junio del 2014).

Como iniciativa propia hicimos volantes donde refleja las transacciones que se puede realizar para dar a conocer más mi negocio (Doña Olga, Pulpería Olga, entrevista 19 de Junio del 2014).

La publicidad que se le da a este nuevo servicio ofrecido por el Banco no es la más adecuada ya que existes personas cercanas al negocio que desconocen los servicios que se ofrecen en el local, es el mismo agente Bancario que se encarga de dar a conocer este servicio, comunicándole a los clientes que lleguen hacer sus compras, las transacciones que pueden realizar desde su local y los beneficios que le genera al ahorrar tiempo y dinero.

La publicidad que genera el Banco al local son rótulos, afiches, volantes y banners lo cual no ha sido lo suficiente para que los clientes se sientan 100\% confiados.
Se observó que los establecimientos que gozan de una publicidad más presentables son los ubicados en las calles centrales como farmacias y agentes de telefonía celular, estos establecimientos tienen una publicidad amplia, banners considerables dentro y fuera del negocio y un espacio muy significativo dentro del establecimiento donde está el agente Banpro con su POS listo para atender a sus clientes Banpro. En los demás establecimientos como pulperías y reposterías la publicidad no es tan buena dentro y fuera del establecimiento, el uso de banners brochurs y mantas no es muy común, el rotulo que el banco les otorga a todos es muy pequeño y no siempre se tiene una buena visibilidad y algunos no se pueden apreciar de una manera correspondiente. (Datos de observación)

De los 360 asociados el 41.19\% representado 201 de las personas utilizan este nuevo servicio por que se ahorran tiempo una profesora nos comentó "por mi trabajo no me queda tiempo ir al banco a realizar mis operaciones por lo cual me facilita a mi estos lugares ya que ellos tienen horario que uno puede visitar a cualquier hora, además los domingos trabajan" (Doña Vanessa Orozco- Seudónimo, Entrevista 16 de Junio del 2014). y un $23.77 \%$ prefieren realizar sus transacciones en los agentes BANPRO porque pagan todos los servicios básicos un señor nos comentó "sería mejor que se pudiera realizar todo lo que se hace en el banco en los agentes bancarios ya que no puedo cambiar cheque, ni retirar remesa" (Don Mauricio Pérez- Seudónimo, Entrevista 18 de Junio del 2014). El $28.89 \%$ dijeron porque el acceso al lugar esta cerca de sus hogares.

Así como un 6.15\% refiriendo otras variables como: horario adecuado, trabaja los Domingos. Otros no le satisface mucho ya que no pueden realizar todas sus operaciones.

Los problemas más frecuentes presentados son los del sistema ya que han tenido diferentes circunstancias en la cual ha generado conflicto y se han solucionado con respecto y educación. 
Según la pregunta si el establecimiento bancario es seguro el $72.78 \%$ representado 262 personas considera que el establecimiento bancario si brinda seguridad. Como iniciativa de algunos establecimientos bancario la Pulpería Olga instalo sus propias oficinas para mayor seguridad del cliente ya que la separo de la venta. Igual que otros establecimientos.

El $27.22 \%$ representado 98 personas sus respuestas fueron que no es seguro. Una señora comento "al establecimiento que visito cuando llego hacer una operación no me gusta ya que la hago frente a las demás personas porque no lo tiene en otro lugar". (Doña Perla Molina- Seudónimo, Entrevista 17 de Junio del 2014).

\section{Infraestructura y condiciones del lugar}

En los diez y ocho establecimientos que se visitaron se observó que el lugar donde se encuentra establecidos los Agentes BANPRO, el 30\% no presentan las condiciones favorables ya que los negocios son grandes y con infraestructura muy pequeña no la adecuada para agregarle este nuevo servicio, el 70\% de estos locales presentan buenas condiciones al ofrecer sus servicios en un lugar amplio y agradable, además estos establecimientos, están bien definidos ya que son lugares donde es muy concurrido y conocido por los usuarios, porque donde están ubicados son puntos clave para la ejecución de este servicio.

La cantidad de personas que visitan estos establecimientos varía según las fechas de pago, debido a que la transacción que tiene más demanda según los agentes es el pago de servicios básicos estos establecimientos realizan más transacciones a final de mes y como negocios de productos básicos tienen una clientela variada y constante, podría decirse que hay una buena afluencia de clientes que podrían utilizar los servicios del agente Banpro al enterarse de su existencia en ese negocio. Se observó que los establecimientos donde están ubicados son casas seguras, aunque los lugares donde están ubicados los Agentes Banpro, no están respaldados por un agente de Seguridad (Datos de observación de los establecimientos bancarios)

\section{Satisfacción de los clientes}

Debido al punto de cada agente Banpro y la afluencia que este recibe el tiempo de cada transacción realizada por cada cliente Banpro varía.

Muchos clientes llegan y son atendidos por el agente y este tarda de 5 a 7 minutos como máximo en cada transacción

Un punto muy importante es con el monto de transacción ya que en algunos establecimientos si llegan depósitos que exceden el monto máximo de transacción el agente dividí el depósito en 2 o 3 transacciones y esa transacción quita más tiempo al cliente, pero es de ganancia para el agente ya que en cada transacción gana más.

Cabe recalcar que sucedió que en algunas transacciones el tiempo de atención fue de 15 minutos como 20 minutos máximo, esto fue debido a que el cliente hizo más de una transacción y esto está sujeto a decisión de cada agente de acuerdo al establecimiento si es ético hacer este tipo de transacciones por cliente, el banco debe revisar de forma minuciosa este tipo de estrategia utilizada por algunos agentes Banpro y decidir si es viable y si conveniente.

La mayor importancia y la estrategia especial del servicio de los agentes Banpro en estos puntos estratégicos es la de minimizar el tiempo y maximizar la afluencia de clientes Banpro, ahorrándoles tiempo en sus transacciones y evitar las grandes filas en el banco y la distancia que se encuentran de él. Así que el tiempo de atención debe de ser la prioridad.

En general de acuerdo a lo observado en las encuestas realizadas a los clientes y las entrevistas a los agentes 
estos tienen un tiempo específico de atención por cada transacción y tiene una duración de 4 a 5 minutos en el $90 \%$ de los agentes Banpro.

\section{Satisfacción del cliente al usar el servicio}

De manera total, todos los agentes Banpro distribuidos en los diferentes puntos de la ciudad de Estelí cumplen con los estándares la atención y explicación del servicio a todos sus clientes en sus transacciones.

La mayoría de estos establecimientos son puntos estratégicos y esto ha llevado una mayor satisfacción a los habitantes y clientes de Banpro por el gran beneficio de poder hacer sus transacciones y pagos de servicios básicos como son el pago de agua, luz y depósitos .

La satisfacción de estos clientes es más que positiva y agradecida, lo cual Evitarse la caminata de más de $1 \mathrm{~km}$ hasta el centro de la ciudad muchas veces bajo el sol o el pago del transporte público que muchas veces resulta muy costoso y aún más evitar las inmensas largas filas de personas en la central es más que satisfactoria para ellos.

Se observó que los clientes se sienten con más seguridad, por lo que los Agentes Banpro se encuentran cerca de sus casas y en su mismo barrio y la seguridad de que están haciendo la transacción como que estuvieran en el banco pues esto ha ayudado a que el cliente vuelva a llegar al agente Banpro.

Según las encuestas realizadas como se refleja en la tabla uno de los principales problemas que tiene la población con los agentes BANPRO es no poder realizar todas

Se me capacito sobre el uso del sistema y el encargado siempre esta disponible para cualquier problema o falla del POS, pero en mi caso no e tenido ningún inconveniente con el servicio o el uso del sistema, obteniendo satisfacción por parte de mis clientes. (Propietario Tienda 3D, entrevista 16 de Junio 2014).

Visita constate del capacitador y el manual de funciones (Karina Gallo, Pulpería Karina, entrevista 16 de Junio 2014.)

Tabla Nº1: Problemas con los agentes BANPRO. Universo: 360 encuestas

\begin{tabular}{lll}
\hline \multicolumn{1}{c}{ Variables } & Frecuencia & \multicolumn{1}{c}{$\%$} \\
\hline No pagar todos los servicios. & 278 & $77.22 \%$ \\
Pago no correspondiente & 20 & $5.56 \%$ \\
Mala atención & 57 & $15.83 \%$ \\
Otros & 5 & $1.39 \%$ \\
Total & 360 & $100 \%$ \\
\hline
\end{tabular}

Fuentes: Datos primarios de la encuestas Junio 2014.

En resumen la personas contestaron que solo se le brinda una capacitación de la utilización del servicio no obstante los encargos de los agentes BANPRO se encuentra en constante seguimiento sobre problema que se presenta con lo mismo.

Los problemas más frecuentes presentados son los del sistema ya que han tenido diferentes circunstancias en la cual ha generado conflicto y se han solucionado con respeto y educación.

Entre los servicios ofrecidos por los agentes BANPRO el más utilizado por los usuarios es el pago de los servicios básicos como vemos reflejado en tabla con un porcentaje de $44.08 \%$ depósitos con un $21.93 \%$. Una señora comento que "Yo lo utilizo solo para pagar servicios básicos” (Doña Juana Cruz-Seudónimo, Entrevista 12 de Junio del 2014). Entre lo menos que se encuentra es el pago de tarjeta con $6.58 \%$ y consulta de saldo con un $5.70 \%$. 
Tabla $\mathbf{N}^{\circ} 2$. Servicios de los agentes BANPRO. Universo: 360 encuestas

\begin{tabular}{lll}
\hline \multicolumn{1}{c}{ Variables } & Frecuencia & \multicolumn{1}{c}{$\%$} \\
\hline Pago de servicio & 201 & $44.08 \%$ \\
Depósitos & 100 & $21.93 \%$ \\
Tempo aire & 99 & $21.71 \%$ \\
Pago de tarjeta & 30 & $6.58 \%$ \\
Consulta de saldo & 26 & $5.70 \%$ \\
Remesas & 0 & $0 \%$ \\
Cambio de cheque. & 0 & $0 \%$ \\
\hline
\end{tabular}

Fuentes: Datos primarios de la encuestas Junio 2014.

Lo que es remesas y cambio de cheque no lo realiza los agentes BANPRO. Según el Banco primero para las remesas necesita una configuración especial para la conexión además algunas agencias del extranjero trabaja con claves que le sería muy difícil trabajarla con loa agentes. Pero se encuentra en prueba piloto acerca de las remesas buscando una forma para que se pueda realizar. Con los cheques no se puede ya que para pagarlo necesita conocimientos básicos, que es fácil de capacitarlo pero no tiene un sistema para que los dueños de los agentes revisen si el cheque tiene fondo o no. Y además entre algunos problemas que se presenta con esta operación por lo que no se puede implementar con los agentes.

Las transacciones que se realizan en estos Agentes Banpro varían en algunos establecimientos se realizan de 4 a 5 transacciones por día aunque en otros establecimientos se dan de 30 a 35 transacciones por día esto se da porque existe mayor afluencia de clientes y puede influir el tiempo que tiene los agentes de prestar este nuevo servicio ya que no tienen el mismo tiempo. Dentro de los servicios de mayor demanda se encuentra el pago de servicios básicos y deposito, estos establecimientos son más visitados por personas que desean un servicio rápido.

Se observó que con la existencia de estos Agentes Banpro los dueños de los negocios le dan mayor publicidad a su establecimiento lo cual se puede decir que es una estrategia que genera mayor demanda de clientes logrando el Banco una mayor satisfacción de clientes e incorporando a personas que quieren tener una mayor experiencia aunque trabajen por cuenta propia pero su representación es el banco y ellos mismos como Agentes Bancarios, por lo cual logran una mayor confiabilidad y respeto de parte de los usuarios. (Datos de observación en los establecimientos bancarios).

\section{Estrategia actual de BANPRO para su Potencialización}

Los diferentes dueños de establecimiento bancarios nos comentaron sobre el medio que utilizo el banco para que ellos se asociara fueron: El banco es quien localiza el negocio mediante un promotor quien nos visitó y explico de lo que trataba el nuevo servicio y los requisitos que se requerían para ser un agente bancario, entre los más relevantes fue que contáramos con disponibilidad de dinero ya que cada transacción que se iba realizar se trabajaría con dinero propio, de igual manera que el negocio estuviese matriculado y fuese conocido y con mucho movimiento, después de cumplir con los requisitos se me crearon dos cuentas, y se me capacito sobre el uso del sistema y aunque no se me volvió a brindar más capacitaciones el encargado de los Agentes Banpro me visita constantemente para verificar no haya algún problema o fallas en el POS. (Dueño de Ferretería Estelí, entrevista 17 de Junio del 2014).

El Banco realizo un sondeo de los establecimientos concurrido siendo claves para la ejecución de este servicio y se verificó si cumple los requisitos como son: negocio propio, capital disponible para realizar las transacciones ya que se trabaja con dinero propio, tener una edad aceptable para poder manejar correctamente la máquina, negocio estuviera matriculado y cumpliendo con los impuestos, se procedió a apertura una cuenta en el Banco, se brindaron las capacitaciones debidas. 
En la tabla No.3 Según los resultados de las encuestas realizadas como la tabla lo refleja el $42.36 \%$ de las personas asociadas comenta que la mejor estrategia para dar a conocer el nuevo servicio de los agentes BANPRO es darle más publicidad por parte del Banco. Así como se comentó anteriormente algunas de las personas conocieron este servicio por los mismos dueños de los establecimientos.

Un $23.40 \%$ respondieron que deben de mejorar el sistema ya que se tarda en cargar algunas operaciones tanto para realizarla como para bajarla del sistema. Un $15.02 \%$ dijeron que es ampliar las opciones disponibles de operaciones pero anteriormente se dejó claro que no se puede porque algunas requieren de mayor supervisión y procesos para realizarla.

Tabla N³. Estrategia que debería aplicar el Banco Universo: 360 encuestas.

\begin{tabular}{llc}
\hline \multicolumn{1}{c}{ Variables } & Frecuencia & \multicolumn{1}{c}{$\%$} \\
\hline Publicidad & 172 & $42.36 \%$ \\
Mejorar el sistema & 95 & $23.40 \%$ \\
Ampliar opciones de servicios & 61 & $15.02 \%$ \\
$\begin{array}{l}\text { Capacitación de atención al } \\
\text { cliente }\end{array}$ & 69 & $17.00 \%$ \\
Otros & 9 & $2.22 \%$ \\
\hline
\end{tabular}

Fuentes: Datos primarios de la encuestas Junio 2014.
Un $17 \%$ dijeron que sería capacitar al personal que maneja este sistema. Pero el encargado de este servicio por parte del Banco comento "no se puede ya que sería un costo que el banco no lo tienen presupuestado, ya que a ellos solo se le capacita de cómo manejar el sistema" (Alcides Ramón Rivera Ruiz, Técnico BANPRO, Entrevista 12 de Junio de1 2014). Un 2.22\% de las personas dijeron otros como que necesitan de un lugar específico en la cual se ubica el área donde se va atender el servicio.

mi única estrategia para atraerlos es diciéndole cuando vienen hacer sus compras las ventajas que tienen de pagar en mi negocio y se sienten más cómodos, aunque he tenido el problema de que los Boucher de luz y teléfono no se me imprimen y se ha tenido que estar llamando al encargado de lo que son estos locales y él tiene llamar a las sucursales para que se imprima el reporte.

\section{Análisis FODA servicio del Agente BANPRO}

En base a la información anterior se realizo un análisis a los agentes BANPRO para identificar las principales fortalezas, debilidades y así mismo detectar las posibles oportunidades y enfrentar las amenazas para su desarrollo.

\section{Fortalezas}

Oportunidades

- Cartera de cliente amplia.

- Producto fiable y disponible.

- Producto de última tecnología de atención al cliente.

- Más punto de atención a los clientes.

- Mayor horario de atención al cliente ajustando por el establecimiento.

- Fácil uso del sistema para realizar las operaciones en los agentes BANPRO.

- Seguimiento y resolución de los problemas que se presenta al realizar algunas operaciones (Servicios básicos).

- Cobertura en otros nichos de mercados.

- Demanda creciente del mercado.

- BANPRO provee del material de publicidad necesario al establecimiento.

- Credibilidad en la institución financiera.

- Beneficio económico obtenido a través del Banco.

- Facilidad en el otorgamiento de productos y servicios para los dueños de los agentes bancarios (otorgamiento préstamo).

- La mayoría de los propietarios tiene un alto nivel académico. 
- Espacio reducido para atender la demanda.

- Falta de capacitaciones del Banco para mejorar la atención a los clientes en estos establecimientos.

- Competencia de otros establecimientos.

- Problemas que se pueda presentar al realizar pago de servicios públicos.

- Reimpresión de voucher.

- Mejorar la utilización de medios publicitarios masivos para atraer nuevos cliente.
- Entrada de nuevos Agentes BANPRO.

- Los agentes están limitados con algunas operaciones que se realizan.

- Mantener estándares de calidad en la atención de clientes en estos establecimientos.

- El negocio se expone a robos al ver que hay bastante influencia por parte de los clientes a realizar operaciones.

\section{Planteamiento MECA del Agente BANPRO}

Para empezar a precisar la estrategia se suele responder al análisis DAFO con las aplicaciones MECA, lo que nos sirve de orientación estratégica:

\section{Mantener las Fortalezas}

- Concientizar en el uso de productos que sustituya el manejo de efectivo.

- Seguir transformando los Producto de tecnología de atención al cliente.

- Mejorar la experiencia del cliente con un trato correcto, profesional y amable.

- Diseñar más sistema fácil para el uso adecuado del usuario.

- Responder a los problemas de la misma manera a la que se ha ido solucionado.

- Tecnificar más a los propietarios de estos establecimientos con seminario no solo de atención de clientes.

\section{Explorar oportunidades}

- Crear y mejorar la estrategia de agrupamiento, según la cual las organizaciones se establecen en las cercanías de sus competidores para aprovechar la propagación de los conocimientos.

- Diseñar un plan de publicidad estándar para todos los agentes BANPRO.

- Para explotar la credibilidad hay que tener mayor contacto con el cliente para que este conozco y se acuerde al momento de utilizar el servicio. permitiendo mejorar la interacción conociendo sus necesidades para poder ofrecerle la solución.

- Como agente bancario hacer uso de las facilidades que pueda tener con el banco para desarrollar más su negocio.

\section{Corregir las Debilidades}

- Diseñar un modelo de ventanilla o cubículo para estos establecimientos

- Diseñar un plan de incentivos para los agentes BANPRO para mejorar la competitividad y estos se esmere para ampliar su cobertura de atención.

- Mejorar el sistema para prevenir los problemas que actualmente presenta estos agentes BANPRO.

- Permitir la reimpresión de voucher.

\section{Afrontar las Amenazas}

- Establecer normas de seguridad que resguarde la seguridad de los clientes y del propio establecimiento. 
Líneas estratégicas para mejorar las estrategias empresariales actuales del servicio Agente BANPRO.

Después de haber establecido el análisis FODA, la estrategia que se propone se basa en la redacción de sus debilidades para mejor las formas de las estrategias actuales ya usada en el nuevo servicio del banco Agente BANPRO.

Línea $N^{\circ}$ 1: Diseño de Publicidad

Línea $\mathbf{N}^{\circ}$ 2: Capacitación a los propietarios de los negocios

Línea $\mathrm{N}^{\circ}$ 3: Condicionamiento de la ubicación del agente BANPRO

\section{CONCLUSIONES}

Al finalizar el presente estudio investigativo en la institución financiera BANPRO, nuestro objetivo fue determinar el efecto de las estrategias empresariales de los Agentes BANPRO, para la propuesta de estrategias en mejora de la satisfacción de los clientes. En base a esto se concluye:

La principal estrategia que utilizaron fue localizar puntos claves donde se encuentran ubicados los Agentes Bancarios, son establecimientos muy reconocidos y con mucha afluencia de clientes, además de esto ampliarse en el mercado y a la vez brindarle un mejor servicio a sus clientes al facilitarle los pagos y dando mas accesibilidad a los usuarios.

Se valoro las ventajas y desventajas de los Agentes Bancarios con respecto a la satisfacción de los clientes, siendo la pricipal ventaja que ofrecen una variedad de transacciones que los clientes pueden realizar cuando lleguen hacer sus compras y cualquier día. Como principal desventaja es la publicidad, no es reconocido por toda la población y la población no esta bien informado de su uso por lo que muestran un poco de inseguridad y prefieren seguir haciendo sus pagos en la sucursal.

Y por ultimo se definieron unas propuestas para mejorar este servicio, y se destacan mejorar la publicidad de este nuevo servicio, para mayor confiabilidad, asistencia y satisfacción de los usuarios.

\section{RECOMENDACIONES}

A fin de que la presente investigación sea de gran utilidad para los Agentes BANPRO, población, Banco, la Faculta Regional Multidisciplinaria, en base a lo planteado en este estudio y la problemática de los efectos del nuevo servicio que ofrece BANPRO, se les recomienda:

A los agentes BANPRO, Establecer este servicio en un lugar específico dentro del mismo local para una mejor ubicación y comodidad. Hacer sentir al cliente seguro y confiable en cada transacción que realice al momento de usar este servicio. Brindar publicidad a cada cliente nuevo que se presente al establecimiento. Así mismo en la utilización del POS, si presenta algunos problemas dar respuesta sin hacer esperar mucho al cliente.

A la población en general, Cada vez que visiten un Agente Banpro deben estar seguros y confiables de que la transacción que realicen es segura. Que hagan uso de este nuevo servicio ya que el banco lo ha implementado con el fin de que los usuarios se les haga más fácil realizar sus transacciones, lo cual les ahorra tiempo y menos costo.

A BANPRO, al momento de ubicar a los Agentes Bancarios verificar estos no estén tan cerca de otros ya que estos causa competitividad y la demanda o asistencia de los clientes es poca. Así mismo establecer nuevas formas de publicidad para que el usuario tenga más conocimiento sobre el uso de este nuevo servicio. 
A la Facultad Regional Multidisciplinaria, que siga creando profesionales de alta calidad, motivándolos a realizar trabajos investigativos innovadores que les ayude en la obtención de conocimientos para su formación profesional.

\section{BIBLIOGRAFIA}

Blandón García, Sergio (2008). Legislación bancaria y Financiera de la república de Nicaragua. $1^{\mathrm{a}}$ ed. Managua, Nicaragua. Grupo Editorial Acento. Pág. 3.Fecha de consulta: 13/04/14
Chiavenato. (2002). Editorial tercera edición por McGRAW-HILL INTERAMERICANA, S.A., Bogotá, Colombia (consultor), Administración. Proceso Administrativo. Tercera edición, pág. 116. Stoner. J. Freeman. E. Gilbert. D. (1996). Administración 6a. Ed. Editorial: PRENTICE HALL HISPANOAMERICANA, S.A. México. Pág. 296.

Schiffman. L. Kanuk. L. (2005). Comportamiento del consumidor. Área: Marketing. 8ta. Edición. Editorial: PEARSON EDUCACION, México. Pág. 14. 